SHS Web of Conferences 7, 01009 (2014)

DOI: $10.1051 /$ shsconf / 20140701009

(C) Owned by the authors, published by EDP Sciences, 2014

\title{
Research on Full Data Planning Stimulation State of Smart Distribution Automation and Stimulation Evaluating System
}

\author{
Chang Qiang, Wang Lei, Chen Yanwei \\ Kaifeng Power Supply Company of Henan Power Company, State Grid, 475000 Kaifeng, China
}

\begin{abstract}
Smart distribution automation is an important part of smart grids. In our country, power distribution network is open looped and radial. To optimize the power distribution network, this paper aims to conduct full data planning in smart distribution automation from the perspective of annual total cost to explore efficient and practical planning algorithm, and provide fundamental basis for development and completion of smart power distribution system.In this paper, firstly, we analyze the proposal background and features of smart distribution automation to prepare for limits and targets in the process of distribution network planning. Then, according to regional characteristics of smart distribution automation, we provide with appliance strategies of multi ant colony algorithm in planning of smart distribution automation and figure out the stimulation branch circuit data of full data planning in certain power supply region after 500 iterations. It turns out that the convergence of algorithm and the result of optimization are good. At last, after evaluating the result of distribution network planning, we make out an index evaluating system and come up with evaluating process of the planning results as well as algorithm principle of AHP, and finally provide fundamental basis for self-cure of smart distribution automation.
\end{abstract}

Keywords. smart distribution automation; ant colony algorithm; data planning; data stimulation; evaluating system

\section{Introduction}

As we all know, safety and stability urban power grids play important roles in development of urban industry and social activities. In researches on urban power grids, proper planning of power girds can help reduce consumable materials and optimize appliance. Thus, people pay universal attention on planning of smart distribution automation. This paper analyzes the proposal background and features of smart distribution automation, and then figure out the target of smart distribution automation and its technological limits. We finally aim to explore efficient planning algorithm and practical evaluating algorithm to provide urban power grids in our country with technological basis.

As for the planning, stimulation and evaluating of smart distribution automation, many scholars try hard to provide with research methods and conclusions to make contributions to the sound development of urban power grids in our country. Li Bin etc. (2009) have discussed features of smart distribution automation and self-cure capability of the protection and control facing smart distribution automation on basis of demand for protection and control system, then came up with design for protection and control system, and finally respectively analyzed the appliance of structure of 
protection ad control system of smart distribution automation, out-of-step separation of micro-grids as well as non-communication protection in researches of smart distribution automation ${ }^{[1]}$; Sun Hongbin etc. (2012) have analyzed main features and functions of smart distribution automation, and make practical discussions on key technologies such as distributed generation of smart distribution automation, micro-grid, communication, advanced sensing, measuring technology and so on ${ }^{[2]}$; Based on traditional ant colony algorithm, Cheng Peng etc. (2013) have come up with multi ant colony collaborative algorithm and accomplish the optimal arrangement of branch circuit of medium voltage distribution network from the perspective of the overall situation which is of guiding significance towards planning of branch circuit of power distribution network ${ }^{[3]}$.

This paper aims to research on full data planning of smart distribution automation based on former researches to figure out fundamental basis for the sound development of smart distribution automation.

\section{The proposal background of smart distribution automation and its features}

Power system planning is an important footstone for development of power grids. However, simple appliance of design paper and oriented power distribution has turned out to be insufficient to meet social demand for electric energy. To scientifically and efficiently dispatch electricity to users, smart distribution automation has been proposed under the following four backgrounds.

\section{Background 1:}

Power grids have become important parts of national comprehensive system of transport as well as a significant link in the chain of national energy industry.

\section{Background 2:}

The development of various fields depends more and more on electric power, at the same time, it also has new demand for reliable power supply and quality of electric power.

\section{Background 3:}

With the globalization, countries in the world are all exploring development mode of power grids and provide with reliable safeguard for efficiency of power grids.

\section{Background 4:}

At present, users have demands for efficient, reliable, safe, clean and interactive power grids.

To solve problems in proposal background of the definition of smart distribution automation, we need to analyze dispatching feature, evaluating feature and stimulation feature in process of smart distribution automation design to provide guidance for scientific planning algorithm, proper stimulation environment and efficient evaluating methods. We will elaborate general targets of smart distribution automation dispatch, factors which should be considered in planning and design, safety assessment and quick stimulation in following paragraphs.

\section{1) Dispatch target}

The target of smart distribution automation is to efficiently integrate the distributed appliance of electric power and realize collaboration and interaction between core businesses in power grids to prepare for improvement in monitoring analysis of power grids, decision-making control and planning capacity. It should target on deepening researches of operation control technology based on wide area state perception and measure and realize quick isolation of malfunctions and quick evaluation of risks. These all need valid reception of new resources with high-capacity and efficiently collaborate and control electric equipment with energy store function.

\section{2) Planning factors}

It needs the appliance of intelligent electric meters with intercommunication function to prepare for distant connection, load disconnection, waveform record and voltage and electricity monitor. It needs to flexibly reconstruct another power supply path and prepare for the intervention of double loop network, bi-radial network and the third power supply point. We should consider protection measures for power distribution system. We can better plan path of power distribution network if the protector can realize adaptive control with the change of network structure. We need consider the access of 
distributed generation, stored energy and micro-grids as well as control of reactive power optimization and power quality.

\section{3) Safety assessment and quick simulation}

Researches on traditional safety assessment and quick stimulation of power system adopt system modeling and malfunctions calculation. It ignores modeling for information communication system and monitoring system. At present, we need to research on information sharing and operation between relative systems. And we can adopt distributed real-time calculation in safety assessment. The expression of assessment and process of operation should be explained by stimulation data. We can realize self-cure of smart distribution automation through quick stimulation, collaborative/selfadaption control and distributed generation.

\section{Stimulation algorithm and results of smart planning of power distribution branch circuit}

Pontes of power distribution network can be seen in Figure 1. The power distribution network is composed of every branch circuit. Under the circumstance which power stations, high voltage transformer stations and main trunk circuit are decided, branch circuit planning is main direction in current smart distribution automation planning and design.

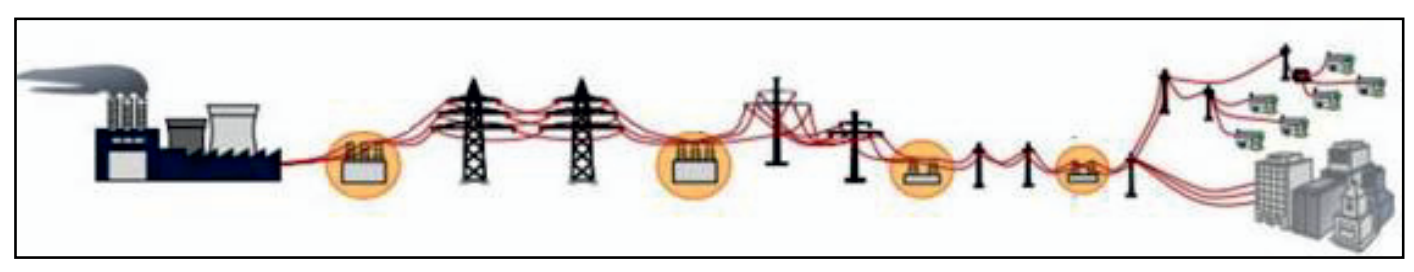

Figure 1. The component of power distribution trunk circuit.

This chapter researches on target of the least annual cost in branch circuit planning. The total cost of branch circuit is composed of investment cost and cost of transmission losses. The target function based on the least cost is shown in formula (1).

$$
f_{\text {min }}=f_{\text {Input }}+f_{\text {Out }}=\left[\underline{r_{0}\left(1+r_{0}\right)^{m}}\right] \cdot \sum_{i=1}^{N} \sum_{j \in J_{i}} l_{i j}+\beta \sum_{i=1}^{N} \sum_{j \in J_{i}} P_{j}^{2} l_{i j}
$$

Based on ant colony algorithm, this chapter will be divided into two parts to elaborate planning strategies of branch circuit in smart distribution automation and results analysis of algorithm stimulation to provide theoretical basis for full data planning of smart distribution automation.

\subsection{Smart planning stimulation strategies of branch circuit of power distribution network based on ant colony algorithm}

The process of power distribution network planning should meet following six limits:

Limit 1 . There should no cross-over phenomenon in power supply regions in process of different circuit planning,

Limit 2. The normal operation of power distribution network is radial.

Limit 3. The power distribution network should supply all users in power supply regions.

Limit 4. The branch circuit in planning is the chosen one, we use existing circuit.

Limit 5. Every circuit has constant maximum load capacity, and its load cannot exceed the maximum one.

Limit 6. Voltage which is access to users should keep its voltage drop in certain range.

Cheng Peng etc. (2013) combine branch circuit with ant colony algorithm, and come up with multi ant colony collaborative algorithm. This algorithm takes every load node as the starting point of ants, 
and takes every node which is on the main trunk circuit which these load nodes belong to as important point. Through ants' constant release and crawling, all ants share the remaining pheromone on the path they pass by. Then we finally figure our optimal arrangement project of branch circuit [3].

When ant $m$ move from node $i$ to adjacent node ${ }^{j}$ at $t$, the state transition probability can be seen in formula (2)

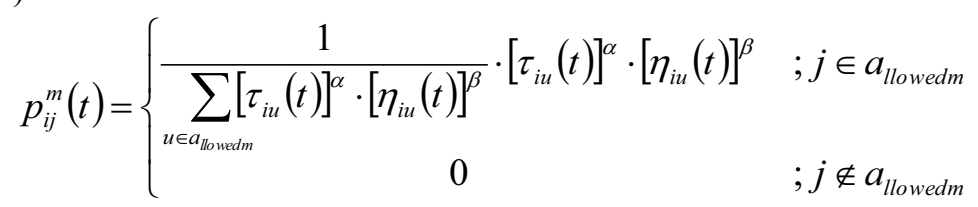

In formula (2), $a_{\text {llowedm }}=\left\{n_{\text {eighbori }}-t_{\text {abum }}\right\}$ represents ant $m$,s next adjacent node which it can take. $\tau_{i j}(t)$ represents information amount on path $(i, j)$ at $t$, while $\alpha, \beta$ represent index with relative importance between pheromone of control and heuristic information. ${ }^{d_{i j}}$ represents distance on path $(i, j)$, and its derivative $\eta_{i j}(t)$ represents heuristic function. Update of pheromone on path $(i, j)$ from $t$ to $t+n$ can be seen in formula (3).

$$
\tau_{i j}(t+n)=(1-\rho) \cdot \tau_{i j}(t)+\Delta \tau_{i j}(t) ; \Delta \tau_{i j}(t)=\sum_{m=1}^{M} \Delta \tau_{i j}^{m}(t)
$$

In formula (3), $(1-\rho)$ represents residual factors of pheromone, while $\rho$ represents volatilized factors of pheromone. $\Delta \tau_{i j}(t)$ represents between time $t_{\sim} t+n$, change of pheromone amount on path $(i, j) . \Delta \tau_{i j}^{m}(t)$ represents the $m$ th ant's remaining pheromone on inherent path $(i, j)$ between $t_{\sim} t+n$. And the remaining pheromone of the $m$ th ant on path $(i, j)$ is specific value of a constant $Q_{\text {and the length of the }} m_{\text {th }}$ ant's crawling. If the ant doesn't pass path $(i, j)$, then the remaining pheromone value is 0 . when ant's crawling accomplishes primary circulation, then take the shortest path $S_{N L}(L O O p)$ of every colony of ants conduct overall update of pheromone.

We can conduct computer stimulation for smart distribution automation planning based on multi ant colony collaborative algorithm according to the process in Figure 2. In the figure, A represents parameter setting, B represents pheromone on every path of initial power distribution network. C represents releasing ant $m$ on very load point in power supply regions. D represents the next adjacent destination which ant $m$ chooses according to state transition probability. E represents whether the length of movement is greater than limited value. F represents every colony of ant $m$ can reach the scale of ant colony $m$. G represents local update of pheromone, and $\mathrm{H}$ represents the sum of shortest path $S\left(L_{\text {oop }}\right)$ which all ants take in the ${ }^{L_{o o p}}$ th circulation.

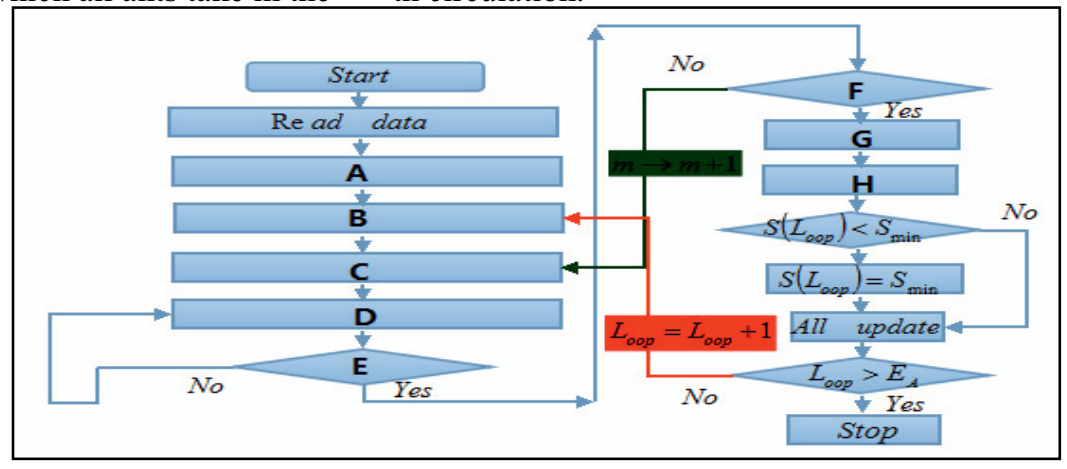

Figure 2. Algorithm process of smart distribution automation planning. 


\subsection{Stimulation results of full data planning in smart distribution automation}

The capacity of high voltage transformer substation we choose is $2 \times 40$ MVA. The type of main trunk circuit in its power supply power is LGJ-240, and that of branch circuit is LGJ-185. We suppose the scale $M$ of each colony of ants is 40 and the initial value of pheromone of every path is 1 , times of iterations are 500. Original data of partial load and branch circuit data in process of optimization can be seen in Table 1.

Table 1. Original data of partial load and branch circuit data in process of optimization.

\begin{tabular}{|c|c|c|c|c|c|}
\hline \multicolumn{3}{|c|}{ Original Data of Partial Load } & \multicolumn{3}{c|}{ Branch Circuit Data in Process of Optimization } \\
\hline $\begin{array}{c}\text { Number of Main } \\
\text { Trunk Circuit }\end{array}$ & $\begin{array}{c}\text { Coordinates of } \\
\text { Load Points }\end{array}$ & $\begin{array}{c}\text { Load } \\
(\mathrm{kW})\end{array}$ & $\begin{array}{c}\text { Number of } \\
\text { Iterations }\end{array}$ & $\begin{array}{c}\text { Total Expenses } \\
\text { (Yuan) }\end{array}$ & $\begin{array}{c}\text { Total Length } \\
\text { (Kilometer) }\end{array}$ \\
\hline 1 & $(1054,1533)$ & 444 & 1 & $1,079,000$ & 34.86 \\
\hline 1 & $(1432,1526)$ & 267 & 2 & $1,049,400$ & 33.22 \\
\hline 1 & $(1807,1536)$ & 330 & 3 & 997,300 & 31.81 \\
\hline 1 & $(1755,1028)$ & 661 & 280 & 783,800 & 25.67 \\
\hline 1 & $(2105,1021)$ & 586 & 281 & 781,300 & 25.3 \\
\hline 1 & $(2640,1654)$ & 331 & 282 & 766,200 & 24.78 \\
\hline 2 & $(2871,1350)$ & 551 & 370 & 661,900 & 21.34 \\
\hline 2 & $(2901,1052)$ & 309 & 371 & 660,800 & 21.33 \\
\hline 2 & $(3118,1383)$ & 712 & 372 & 660,000 & 21.32 \\
\hline 2 & $(3085,1675)$ & 137 & 488 & 660,000 & 21.32 \\
\hline 2 & $(3481,1683)$ & 365 & 499 & 660,000 & 21.32 \\
\hline 2 & $(3802,1382)$ & 268 & 500 & 660,000 & 21.32 \\
\hline
\end{tabular}

According to results of stimulation data above, the appliance of multi ant colony collaborative algorithm have strong convergence and 500 iterations meet the demand for data optimization.

\section{Evaluating system of distribution network planning}

\subsection{Comprehensive Evaluating Index System of Distribution Network}

To evaluating smart distribution automation, this paper has chosen five first-class indexes and twelve second-class indexes. The first-class indexes are S1-power supply safety, S2-power supply reliability, S3-economical efficiency, S4-adaptation, S5-harmony, these first-class indexes which we talk above corresponds to following second-class indexes. They are S11- $(N-1)$ verification, S12- counterforce to power failure in large areas, S21-average interruption duration of system, S22-average interruption frequency of system, S23-average interruption duration of users, S24-average power supply availability of system, S31-operating economy, S32-construction economy, S41-resources margin, S42-power supply capacity margin, S43-power grids extension margin, S51-capacity coordination of high and medium voltage power distribution network, S52-power supply capacity matching rate of high and medium network, and S53-load balance degree. Then we can build index system which takes comprehensive evaluating value of T-smart distribution automation as target layer. Figure 3 shows the comprehensive evaluating index system of smart distribution automation. 


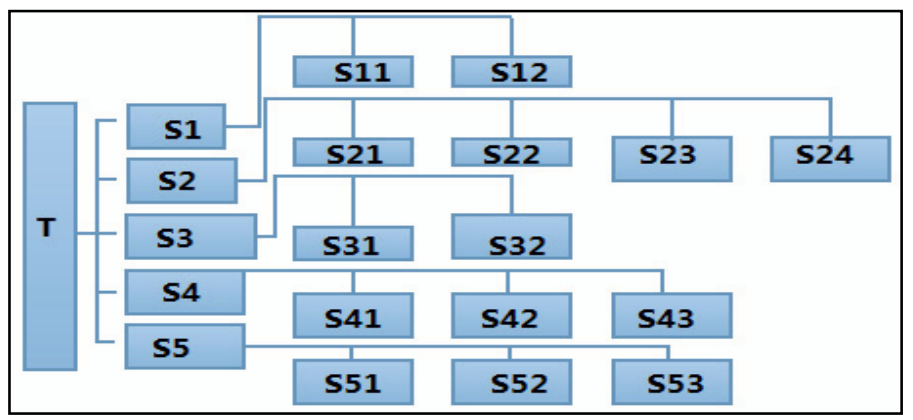

Figure 3. Comprehensive evaluating index system structure of smart distribution automation.

\subsection{Processes of Evaluating of Distribution Network Planning}

Evaluate the results of branch circuit planning of smart distribution automation, that is, evaluate the index system in Figure 3. Figure 4 shows the evaluating processes, and paraphrase of numbers in Figure 4 has been shown in Table 2.

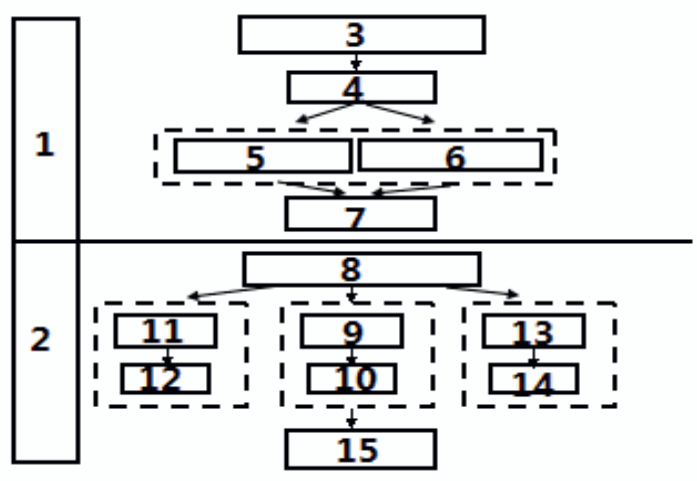

Figure 4. Processes of distribution network planning evaluating.

Table 2. Paraphrase of numbers in figure of processes of distribution network planning evaluating.

\begin{tabular}{|c|l|c|l|}
\hline No. & Paraphrase & No. & Paraphrase \\
\hline 1 & Setting phase of evaluating index & 9 & Single index evaluating of current power grids \\
\hline 2 & Planning evaluating phase & 10 & Comprehensive grades of current power grids \\
\hline 3 & $\begin{array}{l}\text { Analysis on features of power supply } \\
\text { regions and load features }\end{array}$ & 11 & $\begin{array}{l}\text { Single index evaluating of smart distribution } \\
\text { automation based on general algorithm }\end{array}$ \\
\hline 4 & Choice evaluation index system & 12 & $\begin{array}{l}\text { Comprehensive grade of smart distribution } \\
\text { automation based on general algorithm }\end{array}$ \\
\hline 5 & Choice index evaluating criteria & 13 & $\begin{array}{l}\text { Single index evaluating of smart distribution } \\
\text { automation based on ant colony algorithm }\end{array}$ \\
\hline 6 & Choice index evaluating standards & 14 & $\begin{array}{l}\text { Comprehensive grade of smart distribution } \\
\text { automation based on ant colony algorithm }\end{array}$ \\
\hline 7 & Setting index weight & 15 & $\begin{array}{l}\text { Comparison and analysis of comprehensive } \\
\text { grade of smart distribution automation planning }\end{array}$ \\
\hline 8 & Planning data input & \multicolumn{2}{|l}{} \\
\hline
\end{tabular}


We adopt AHP to evaluate distribution network planning, as it shows in formula (4):

$$
S^{(k+1)}=\sum_{j=1}^{n} S_{j}^{(k)} W_{j}^{(k)}
$$

In formula (4), $S^{(k+1)}$ represents the grades of certain property $A^{(k+1)}$ in the $\mathrm{k}+1$ layer of hierarchical structure in index evaluating system. ${ }^{n}$ represents the number of sub-property in $k$ layer of property $A^{(k+1)}$. While $S_{j}^{(k)}$ represents grades on sub-property $j$ in the $k$ layer of property $A^{(k+1)}$, and ${ }^{W_{j}^{(k)}}$ represents the weight of sub-property ${ }^{j}[5]$.

\section{Conclusion}

This paper elaborately analyzes planning principles and algorithm of smart distribution automation and apply high-voltage transformer substation which capacity is $2 \times 40 \mathrm{MVA}$ to optimize the planning. Then it figure out the multi ant colony algorithm is of good convergence and optimization through information from branch circuit data. Otherwise, it builds comprehensive evaluating index system of smart distribution automation and provides with AHP evaluating and analysis process of index evaluating system.

Advantages and disadvantages of planning algorithm matter a lot for operation of smart distribution automation. To accelerate the development of power distribution network, it's necessary to introduce new algorithm. The development of computing technology and software technology provides basis for realization of complex algorithm. Thus, the intelligent planning algorithm in this paper can also adopt BP neural network algorithm and many other advanced intelligent algorithms.

\section{References}

1. Li Bin etc.. Design and Research on Protection and Control of Smart Distribution Automation [J]. Proceedings of the CSEE. 2009.29:1-6.

2. Sun Hongbin etc.. Smart Distribution Automation System Design and Research [J]. China Rural Water Resources and Hydropower. 2012(2):131-134.

3. Cheng Peng etc.. Branch Circuit Intelligent Planning of Medium Voltage Distribution Network Based on Multi Ant Colony Collaborative Algorithm [J]. Power System Technology. 2013.37(7):1936-1940.

4. Li Zhenkun etc.. Real-time Evaluating Analysis on Power Supply Capacity of Power Distribution Network [J]. Power System Automation. 2009.33 (6):36-40.

5. Xiao Jun etc. Comprehensive Evaluating Index System and Methods of Power Distribution Network Planning [J]. Power System Automation. 2008.32 (15):36-40.

6. Yu Yixin etc.. Quick Stimulation of Smart Power Grids and Optimizing Methods of dispatching in Stimulation Tasks [J]. Computer Engineering and Appliance. 2009.45 (19):26-30. 